\title{
Certain results on Euler-type integrals and their applications
}

R. Jabee

M. Shadab

R.B. Paris

This is a post-peer-review, pre-copyedit version of an article published in Ramanujan journal. The final authenticated version is available online at: http://dx.doi.org/10.1007/s11139-019-00238-w 


\title{
Certain results on Euler-type integrals and their applications
}

\author{
S. JABEE ${ }^{a}$, M. SHADAB ${ }^{a *}$ AND R.B. PARIS ${ }^{b \dagger}$ \\ ${ }^{a}$ Department of Applied Sciences and Humanities, \\ Faculty of Engineering and Technology, \\ Jamia Millia Islamia University, New Delhi 110025, India \\ ${ }^{b}$ Division of Computing and Mathematics, Abertay University, \\ Dundee DD1 $1 H G, U K$.
}

\begin{abstract}
This paper deals with the evaluation of some definite Euler-type integrals in terms of the Wright hypergeometric function. We obtain a theorem on the Wright hypergeometric function and then use this theorem to evaluate some definite integrals. Further, we derive some results as applications of these evaluations. Multi-variable cases of the derived results of this paper are also briefly discussed.
\end{abstract}

MSC: 33C20, 33E12, 33C05, 33B99

Keywords: Euler-type integrals; Wright hypergeometric function; Mittag-Leffler function; generating function.

\section{Introduction}

The generalization of the generalized hypergeometric series ${ }_{p} F_{q}$ is due to Fox $[7]$ and Wright $[30,31]$ who studied the asymptotic expansion of the so-called Wright hypergeometric function defined by (see $[27$, p. 21])

$$
{ }_{p} \Psi_{q}\left[\begin{array}{c}
\left(\alpha_{1}, A_{1}\right), \ldots,\left(\alpha_{p}, A_{p}\right) ; z \\
\left(\beta_{1}, B_{1}\right), \ldots,\left(\beta_{q}, B_{q}\right) ;
\end{array}\right]=\sum_{k=0}^{\infty} \frac{\prod_{j=1}^{p} \Gamma\left(\alpha_{j}+A_{j} k\right)}{\prod_{j=1}^{q} \Gamma\left(\beta_{j}+B_{j} k\right)} \frac{z^{k}}{k !} .
$$

The coefficients $A_{j} \in \mathbf{R}^{+}(j=1, \ldots, p)$ and $B_{j} \in \mathbf{R}^{+}(j=1, \ldots, q)$ are such that

$$
1+\sum_{j=1}^{q} B_{j}-\sum_{j=1}^{p} A_{j} \geq 0
$$

Here and in the following, let $\mathbf{C}, \mathbf{R}, \mathbf{R}^{+}, \mathbf{Z}$ and $\mathbf{N}$ be the sets of complex numbers, real numbers, positive real numbers, integers and positive integers, respectively, and let

$$
\mathbf{R}_{0}^{+}:=\mathbf{R}^{+} \cup\{0\}, \quad \mathbf{N}_{0}:=\mathbf{N} \cup\{0\} \quad \text { and } \quad \mathbf{Z}_{0}^{-}:=\mathbf{Z} \backslash \mathbf{N} .
$$

\footnotetext{
* Corresponding author: M. Shadab

${ }^{\dagger}$ E-mail: saimajabee007@gmail.com (S. Jabee), shadabmohd786@gmail.com (M. Shadab), r.paris@abertay.ac.uk (R.B. Paris)
} 
For convenience in presentation we also introduce suitable normalizing factors into the definition in (1.1) and define the normalized Wright function

$$
{ }_{p} \hat{\mathbf{\Psi}}_{q}\left[\begin{array}{c}
\left(\alpha_{1}, A_{1}\right), \ldots,\left(\alpha_{p}, A_{p}\right) ; \\
\left(\beta_{1}, B_{1}\right), \ldots,\left(\beta_{q}, B_{q}\right) ;
\end{array}\right]=\sum_{k=0}^{\infty} \frac{\prod_{j=1}^{p} \Gamma\left(\alpha_{j}+A_{j} k\right) / \Gamma\left(\alpha_{j}\right)}{\prod_{j=1}^{q} \Gamma\left(\beta_{j}+B_{j} k\right) / \Gamma\left(\beta_{j}\right)} \frac{z^{k}}{k !}
$$

A special case is

$$
\begin{gathered}
{ }_{p} \hat{\Psi}_{q}\left[\begin{array}{c}
\left(\alpha_{1}, 1\right), \ldots,\left(\alpha_{p}, 1\right) ; z \\
\left(\beta_{1}, 1\right), \ldots,\left(\beta_{q}, 1\right) ;
\end{array}\right]={ }_{p} F_{q}\left[\begin{array}{c}
\alpha_{1}, \ldots, \alpha_{p} ; z \\
\beta_{1}, \ldots, \beta_{q} ;
\end{array}\right] \\
=\sum_{n=0}^{\infty} \frac{\left(\alpha_{1}\right)_{n} \cdots\left(\alpha_{p}\right)_{n}}{\left(\beta_{1}\right)_{n} \cdots\left(\beta_{q}\right)_{n}} \frac{z^{n}}{n !}
\end{gathered}
$$

where $(a)_{k}$ is the Pochhammer symbol or rising factorial defined by $(a)_{n}=\Gamma(a+n) / \Gamma(a)=$ $a(a+1) \ldots(a+n-1)$. Here an empty product is interpreted as 1 . We assume that the variable $z$, the numerator parameters $\alpha_{1}, \ldots, \alpha_{p}$, and the denominator parameters $\beta_{1}, \ldots, \beta_{q}$ take on complex values, provided that no zeros appear in the denominator of (1.4), that is

$$
\beta_{j} \in \mathbf{C} \backslash \mathbf{Z}_{0}^{-} ; j=1, \ldots, q
$$

For more details of ${ }_{p} F_{q}$ including its convergence, its various special and limiting cases, and its further diverse generalizations, see, for example, $[2,19,26]$.

The beta function $B(\alpha, \beta)$ is defined by

$$
B(x, y)= \begin{cases}\int_{0}^{1} t^{x-1}(1-t)^{y-1} d t & (\Re(x)>0 ; \Re(y)>0) \\ \frac{\Gamma(x) \Gamma(y)}{\Gamma(x+y)} & \left(x, y \in \mathbf{C} \backslash \mathbf{Z}_{0}^{-}\right) .\end{cases}
$$

Let us recall the well-known integral representation of the ${ }_{2} F_{1}$ function given by (see, e.g., [19, p. $85]$ and $[26$, p. 65])

$$
\begin{gathered}
{ }_{2} F_{1}(a, b ; c ; z)=\frac{1}{B(b, c-b)} \int_{0}^{1} t^{b-1}(1-t)^{c-b-1}(1-z t)^{-a} d t \\
(\Re(c)>\Re(b)>0, \arg (1-z)<\pi) .
\end{gathered}
$$

A number of extensions of some familiar special functions have been recently investigated (see, for example, $[3,4,5,6,14,22])$. Chaudhry et al. [3] presented an extension of the beta function as follows

$$
\begin{gathered}
B(x, y ; p)=\int_{0}^{1} t^{x-1}(1-t)^{y-1} \exp \left(-\frac{p}{t(1-t)}\right) d t \\
(\Re(p)>0 ; \quad p=0, \Re(x)>0, \Re(y)>0)
\end{gathered}
$$

and showed that this extension has certain connections with the Macdonald, error and Whittaker functions.

Chaudhry et al. [4] used $B(x, y ; p)$ to extend the hypergeometric and the confluent hypergeometric functions, respectively, as follows:

$$
F_{p}(a, b ; c ; z)=\sum_{n=0}^{\infty} \frac{(a)_{n} B(b+n, c-b ; p)}{B(b, c-b)} \frac{z^{n}}{n !}
$$




$$
\left(p \in \mathbf{R}_{0}^{+},|z|<1, \Re(c)>\Re(b)>0\right)
$$

and

$$
\begin{gathered}
\Phi_{p}(b ; c ; z)=\sum_{n=0}^{\infty} \frac{B(b+n, c-b ; p)}{B(b, c-b)} \frac{z^{n}}{n !} \\
\left(p \in \mathbf{R}_{0}^{+}, \Re(c)>\Re(b)>0\right) .
\end{gathered}
$$

The following integral representations are recalled ([4, Eq. (3.2) and Eq. (3.6)])

$$
\begin{gathered}
F_{p}(a, b ; c ; z)=\frac{1}{B(b, c-b)} \int_{0}^{1} t^{b-1}(1-t)^{c-b-1}(1-z t)^{-a} \exp \left(-\frac{p}{t(1-t)}\right) d t \\
\left(p \in \mathbf{R}^{+} ; p=0,|\arg (1-z)|<\pi, \Re(c)>\Re(b)>0\right)
\end{gathered}
$$

and

$$
\begin{gathered}
\Phi_{p}(b ; c ; z)=\frac{1}{B(b, c-b)} \int_{0}^{1} t^{b-1}(1-t)^{c-b-1} \exp \left(z t-\frac{p}{t(1-t)}\right) d t \\
\left(p \in \mathbf{R}^{+} ; \quad p=0, \Re(c)>\Re(b)>0\right) .
\end{gathered}
$$

The Mittag-Leffler function is defined by ([13, p. 261], see also [8, p. 17, Eq. (3.1.1)] and $[11,12])$

$$
E_{\alpha}(z)=\sum_{n=0}^{\infty} \frac{z^{n}}{\Gamma(\alpha n+1)} \quad\left(\alpha \in \mathbf{R}_{0}^{+}, z \in \mathbf{C}\right)
$$

from which it is obvious that

$$
E_{0}(z)=\frac{1}{1-z}(|z|<1), \quad E_{1}(z)=e^{z} \quad \text { and } \quad E_{2}(z)=\cosh z^{1 / 2} .
$$

The Euler-type integrals have the general integrand

$$
u^{a-1}(1-u)^{b-1} f(u) .
$$

The evaluation of such integrals is relevant to many reduction formulas for hypergeometric functions that generalize the evaluation of some symmetric Euler-type integrals implicit by the result contained in [15]. Motivated by the work of Shadab et al. [23] and Ismail and Pitman [9], we evaluate some Euler-type integrals associated with the Mittag-Leffler function $E_{\lambda}[p \xi(t)]$ of the form

$$
\int_{a}^{b}(t-a)^{\alpha-1}(b-t)^{\beta-1}[\chi(t)]^{\gamma} E_{\lambda}[p \xi(t)] d t
$$

for complex parameter $p$ and some particular choices of the functions $\chi(t)$ and $\xi(t)$.

\section{Some evaluations of Euler-type integrals}

In this section, we derive some theorems on the evaluation of the Euler-type integrals

$$
I_{a, b, \lambda}^{\alpha, \beta, \gamma}[\chi(t), \xi(t) ; p]=\frac{1}{B(\alpha, \beta)} \int_{a}^{b}(t-a)^{\alpha-1}(b-t)^{\beta-1}[\chi(t)]^{\gamma} E_{\lambda}[p \xi(t)] d t
$$

for some specific functions $\chi(t)$ and $\xi(t)$. In all cases considered $p \in \mathbf{C}$ is a complex variable. 
Theorem 1. For $a=0, b=\gamma=1, \xi(t)=t(1-t)$ and $\chi(t)=\left(1-x_{1} t\right)^{-\alpha_{1}}\left(1-x_{2} t\right)^{-\alpha_{2}}$, we have the following integral

$$
\begin{aligned}
& I_{0,1, \lambda}^{\alpha, \beta, 1}\left[\left(1-x_{1} t\right)^{-\alpha_{1}}\left(1-x_{2} t\right)^{-\alpha_{2}}, t(1-t) ; p\right] \\
& =\sum_{m, n=0}^{\infty} \frac{(\alpha)_{m+n}\left(\alpha_{1}\right)_{m}\left(\alpha_{2}\right)_{n} x_{1}^{m} x_{2}^{n}}{(\alpha+\beta)_{m+n} m ! n !}{ }_{3} \hat{\Psi}_{2}\left[\begin{array}{r}
(\alpha+m+n, 1),(\beta, 1),(1,1) ; \\
p \\
(\alpha+\beta+m+n, 2),(1, \lambda) ;
\end{array}\right]
\end{aligned}
$$

for $\Re(\alpha), \Re(\beta)>0, \lambda \geq 0$ and $\left|x_{1}\right|,\left|x_{2}\right|<1$, where we recall that ${ }_{p} \hat{\Psi}_{q}$ is the normalized Wright function defined in (1.3).

Proof. We have [9, p. 962(7)]

$$
\int_{0}^{1} t^{\alpha-1}(1-t)^{\beta-1}\left(1-x_{1} t\right)^{-\alpha_{1}}\left(1-x_{2} t\right)^{-\alpha_{2}} d t=B(\alpha, \beta) F_{1}\left(\alpha ; \alpha_{1}, \alpha_{2} ; \alpha+\beta ; x_{1}, x_{2}\right)
$$

$$
\left(\Re(\alpha), \Re(\beta)>0 ; \lambda \geq 0 ;\left|x_{1}\right|,\left|x_{2}\right|<1\right),
$$

where $F_{1}$ is the Appell double hypergeometric series defined by [13, p. 413]

$$
F_{1}\left(\alpha ; \beta, \beta^{\prime} ; \gamma ; x, y\right)=\sum_{m, n=0}^{\infty} \frac{(\alpha)_{m+n}(\beta)_{m}\left(\beta^{\prime}\right)_{n}}{(\gamma)_{m+n} m ! n !} x^{m} y^{n} \quad(|x|,|y|<1) .
$$

Then we set $a=0, b=\gamma=1, \xi(t)=t(1-t), \chi(t)=\left(1-x_{1} t\right)^{-\alpha_{1}}\left(1-x_{2} t\right)^{-\alpha_{2}}$ in $(2.1)$ and use the series expansion for $E_{\lambda}[p t(1-t)]$ in (1.16). After an interchange of the order of integration and summation and use of the above integral followed by simplification using the properties of the gamma function, we obtain (2.2).

We note that ${ }_{3} \hat{\Psi}_{2}(0)=1$ when $p=0$ and the right-hand side of (2.2) reduces to

$$
F_{1}\left(\alpha ; \alpha_{1}, \alpha_{2} ; \alpha+\beta ; x_{1}, x_{2}\right)
$$

in accordance with $(2.3)$.

Theorem 2. For $a=0, b=\gamma=1, \xi(t)=t(1-t)$ and $\chi(t)=\left(1-x_{1} t\right)^{-\alpha_{1}}\left(1-x_{2}(1-t)\right)^{-\alpha_{2}}$, we have the following integral

$$
\begin{array}{r}
I_{0,1, \lambda}^{\alpha, \beta, 1}\left[\left(1-x_{1} t\right)^{-\alpha_{1}}\left(1-x_{2}(1-t)\right)^{-\alpha_{2}}, t(1-t) ; p\right] \\
=\sum_{m, n=0}^{\infty} \frac{(\alpha)_{m}(\beta)_{n}\left(\alpha_{1}\right)_{m}\left(\alpha_{2}\right)_{n} x_{1}^{m} x_{2}^{n}}{(\alpha+\beta)_{m+n} m ! n !}{ }_{3} \hat{\Psi}_{2}\left[\begin{array}{c}
(\alpha+m, 1),(\beta+n, 1),(1,1) ; \\
(\alpha+\beta+m+n, 2),(1, \lambda) ;
\end{array}\right]
\end{array}
$$

$$
\left(\Re(\alpha), \Re(\beta)>0 ; \lambda \geq 0 ;\left|x_{1}\right|,\left|x_{2}\right|<1\right) .
$$

Proof. We have [27, p. 279(17)]

$$
\begin{gathered}
\int_{0}^{1} t^{\alpha-1}(1-t)^{\beta-1}\left(1-x_{1} t\right)^{-\alpha_{1}}\left(1-x_{2}(1-t)\right)^{-\alpha_{2}} d t=B(\alpha, \beta) F_{3}\left(\alpha, \beta, \alpha_{1}, \alpha_{2} ; \alpha+\beta ; x_{1}, x_{2}\right) \\
\left(\Re(\alpha), \Re(\beta)>0 ; \lambda \geq 0 ;\left|x_{1}\right|,\left|x_{2}\right|<1\right),
\end{gathered}
$$

where $F_{3}$ is the Appell double hypergeometric series defined by [13, p. 413]

$$
F_{3}\left(\alpha, \alpha^{\prime} ; \beta, \beta^{\prime} ; \gamma ; x, y\right)=\sum_{m, n=0}^{\infty} \frac{(\alpha)_{m}\left(\alpha^{\prime}\right)_{n}(\beta)_{m}\left(\beta^{\prime}\right)_{n}}{(\gamma)_{m+n} m ! n !} x^{m} y^{n} \quad(|x|,|y|<1)
$$

Then on setting $a=0, b=\gamma=1, \xi(t)=t(1-t)$ and $\chi(t)=\left(1-x_{1} t\right)^{-\alpha_{1}}\left(1-x_{2}(1-t)\right)^{-\alpha_{2}}$ in (2.1), using (1.16) and the above integral, we obtain upon simplification (2.4). 
Theorem 3. For $\chi(t)=u t+v$ and $\xi(t)=(t-a)(b-t)$, we have the following integral

$$
\begin{array}{r}
I_{a, b, \lambda}^{\alpha, \beta, \gamma}[u t+v,(t-a)(b-t) ; p]=\sum_{m=0}^{\infty} \frac{(-\gamma)_{m}(\alpha)_{m}}{(\alpha+\beta)_{m} m !}\left(\frac{-u(b-a)}{a u+v}\right)^{m} \\
\times{ }_{3} \hat{\Psi}_{2}\left[\begin{array}{c}
(\alpha+m, 1),(\beta, 1),(1,1) ; \\
p \\
(\alpha+\beta+m, 2),(1, \lambda) ;
\end{array}\right] \\
\left(\Re(\alpha), \Re(\beta)>0 ; \lambda \geq 0 ;\left|\arg \left(\frac{b u+v}{a u+v}\right)\right|<\pi ; a \neq b\right) .
\end{array}
$$

Proof. From [17, p. 263], we have

$$
\begin{gathered}
\int_{a}^{b}(t-a)^{\alpha-1}(b-t)^{\beta-1}(u t+v)^{\gamma} d t=B(\alpha, \beta){ }_{2} F_{1}\left(-\gamma, \alpha ; \alpha+\beta ;-\frac{(b-a) u}{a u+v}\right) \\
\left(\Re(\alpha), \Re(\beta)>0 ; \lambda \geq 0 ;\left|\arg \left(\frac{b u+v}{a u+v}\right)\right|<\pi ; a \neq b\right) .
\end{gathered}
$$

On setting $\chi(t)=u t+v$, and $\xi(t)=(t-a)(b-t)$ in (2.1), using (1.16) and the above integral and simplifying, we obtain (2.6).

Theorem 4. For $\chi(t)=b-a+\nu(t-a)+\mu(b-t), \xi(t)=(t-a)(b-t) / \chi^{2}(t)$ and $\gamma=-(\alpha+\beta)$, we have following integral

$$
\begin{gathered}
I_{a, b, \lambda}^{\alpha, \beta,-(\alpha+\beta)}\left[b-a+\nu(t-a)+\mu(b-t), \frac{(t-a)(b-t)}{(b-a+\nu(t-a)+\mu(b-t))^{2}}\right] \\
=\frac{(\nu+1)^{-\alpha}(\mu+1)^{-\beta}}{(b-a)} \hat{\Psi}_{2}\left[\begin{array}{c}
(\alpha, 1),(\beta, 1),(1,1) ; \\
(\alpha+\beta, 2),(1, \lambda) ;
\end{array}\right] \\
(\Re(\alpha), \Re(\beta)>0 ; \lambda \geq 0 ; \chi(t) \neq 0 ; a \neq b) .
\end{gathered}
$$

Proof. From [28, p. 261(3.1)] we have

$$
\begin{gathered}
\int_{a}^{b} \frac{(t-a)^{\alpha-1}(b-t)^{\beta-1}}{(b-a+\nu(t-a)+\mu(b-t))^{\alpha+\beta}} d t=B(\alpha, \beta) \frac{(\nu+1)^{-\alpha}(\mu+1)^{-\beta}}{b-a} \\
(\Re(\alpha), \Re(\beta)>0 ; \lambda \geq 0 ; b-a+\nu(t-a)+\mu(b-t) \neq 0 ; a \neq b) .
\end{gathered}
$$

On setting $\chi(t)=b-a+\nu(t-a)+\mu(b-t), \xi(t)=(t-a)(b-t) / \chi^{2}(t)$ and $\gamma=-(\alpha+\beta)$ in equation (2.1), using (1.16) and the above integral and simplifying, we obtain (2.7).

\section{An integral associated with a generating function}

Let us consider a two-variable generating function $G(x, t)$ which can be expanded in a formal power series of $t$ such that

$$
G(x, t)=\sum_{n=0}^{\infty} c_{n} g_{n}(x) t^{n},
$$

where the coefficient set $\left\{c_{n}\right\}_{n=0}^{\infty}$ may contain the parameters of the set $\left\{g_{n}(x)\right\}_{n=0}^{\infty}$ but is independent of $x$ and $t$. 
Theorem 5. Let the generating function $G\left(x, t u^{\delta}(1-u)^{\omega}\right)$ be uniformly convergent under the conditions $u \in(0,1), \delta, \omega \geq 0$ and $\delta+\omega>0$, with $G(x, t)$ defined by (3.1). Then

$$
\begin{aligned}
& \int_{0}^{1} u^{r-1}(1-u)^{s-r-1} G\left(x, t u^{\delta}(1-u)^{\omega}\right) E_{\lambda}[p u(1-u)] d u \\
&=\sum_{n=0}^{\infty} c_{n} g_{n}(x) t^{n}{ }_{3} \Psi_{2}\left[\begin{array}{rr}
(r+\delta n, 1),(s-r+\omega n, 1),(1,1) ; & \\
(s+\delta n+\omega n, 2),(1, \lambda) ; & p
\end{array}\right]
\end{aligned}
$$

for $s>r>0, \lambda \geq 0, p \in \mathbf{C}$, where we recall that ${ }_{p} \Psi_{q}$ is the un-normalized Wright function defined in (1.1).

Proof. On using the definition of generating function $G(x, t)$ given in (3.1) in the left-hand side of (3.2), we obtain

$$
\begin{aligned}
& \sum_{n=0}^{\infty} c_{n} g_{n}(x) t^{n} \int_{0}^{1} u^{r+\delta n-1}(1-u)^{s-r+\omega n-1} E_{\lambda}[p u(1-u)] d u \\
= & \sum_{n=0}^{\infty} c_{n} g_{n}(x) t^{n} \sum_{k=0}^{\infty} \frac{p^{k}}{\Gamma(1+\lambda k)} \int_{0}^{1} u^{r+\delta n+k-1}(1-u)^{s-r+\omega n+k-1} d u .
\end{aligned}
$$

Evaluation of the integral as a beta function then yields the right-hand side of (3.2).

Corollary 1. From (3.2), we obtain

$$
\begin{aligned}
& \int_{0}^{1} u^{r-1}(1-u)^{r-1} G\left(x, t(u(1-u))^{\omega}\right) E_{\lambda}[p u(1-u)] d u \\
&=\sum_{n=0}^{\infty} c_{n} g_{n}(x) t^{n}{ }_{3} \Psi_{2}\left[\begin{array}{rr}
(r+\omega n, 1),(r+\omega n, 1),(1,1) ; & \\
(2 r+2 \omega n, 2),(1, \lambda) ;
\end{array}\right] .
\end{aligned}
$$

Proof. On setting $s=2 r$ and $\delta=\omega$ in (3.2), we obtain (3.3).

We now derive some explicit evaluations of definite integrals in terms of Wright hypergeometric functions by making use of Theorem 5 and consideration of the following generating functions.

Example 3.1 Let us consider the generating function [28, p. 44(8)]

$$
G(x, t)=(1-x t)^{-a}=\sum_{n=0}^{\infty}(a)_{n} \frac{(x t)^{n}}{n !}={ }_{1} F_{0}\left[\begin{array}{c}
a ; \\
-;
\end{array}\right] \quad(|x t|<1) .
$$

Use of this generating function (with $x=1$ ) and Theorem 5 yields

$$
\begin{aligned}
&\left.\int_{0}^{1} u^{r-1}(1-u)^{s-r-1}\left[1-t u^{\delta}(1-u)^{\omega}\right)\right]^{-a} E_{\lambda}[p u(1-u)] d u \\
&=\sum_{n=0}^{\infty} \frac{(a)_{n} t^{n}}{n !}{ }_{3} \Psi_{2}\left[\begin{array}{rr}
(r+\delta n, 1),(s-r+\omega n, 1),(1,1) ; & \\
(s+\delta n+\omega n, 2),(1, \lambda) ;
\end{array}\right]
\end{aligned}
$$

for $s>r>0, \lambda \geq 0, \delta, \omega \geq 0, \delta+\omega>0$

Example 3.2 Let us consider the generating function [18, p.409(2)]

$$
G(x, t)=\sum_{n=0}^{\infty} \frac{(a)_{n}}{(b)_{n}}{ }_{1} F_{1}\left[\begin{array}{l}
a ; \\
b+n ;
\end{array}\right] \frac{t^{n}}{n !}=\Phi_{2}[a, a ; b ; x, t] \quad(|x|,|t|<\infty),
$$


where $\Phi_{2}$ is Humbert's confluent hypergeometric series in two variables defined by [28]

$$
\Phi_{2}\left[b_{1}, b_{2} ; c ; x, y\right]=\sum_{m, n=0}^{\infty} \frac{\left(b_{1}\right)_{m}\left(b_{2}\right)_{n} x^{m} y^{n}}{(c)_{m+n} m ! n !} \quad(|x|,|y|<\infty) .
$$

Use of this generating function and Theorem 5 yields

$$
\begin{aligned}
&\left.\int_{0}^{1} u^{r-1}(1-u)^{s-r-1} \Phi_{2}\left[a, a ; b ; x, t u^{\delta}(1-u)^{\omega}\right)\right] E_{\lambda}[p u(1-u)] d u \\
&=\sum_{n=0}^{\infty} \frac{(a)_{n}}{(b)_{n}} \frac{t^{n}}{n !}{ }_{1} F_{1}\left[\begin{array}{c}
a ; \\
b+n ;
\end{array}\right]{ }_{3} \Psi_{2}\left[\begin{array}{rr}
(r+\delta n, 1),(s-r+\omega n, 1),(1,1) ; & p \\
(s+\delta n+\omega n, 2),(1, \lambda) ;
\end{array}\right]
\end{aligned}
$$

for $s>r>0, \lambda \geq 0, \delta, \omega \geq 0, \delta+\omega>0,|x|<\infty$.

Example 3.3 Let us consider the generating function [19, p.276(1)]

$$
G(x, t)=\left(1-2 x t+t^{2}\right)^{-a}=\sum_{n=0}^{\infty} C_{n}^{(a)}(x) t^{n} \quad(|x t|<1),
$$

where $C_{n}^{(a)}(x)$ denotes the Gegenbauer or ultraspherical polynomial [19]. Use of this generating function (with $x=1$ ), Theorem 5 and the fact that $C_{n}^{(a)}(1)=(2 a)_{n} / n$ ! [13, Eq. (18.5.9)], then yields

$$
\begin{aligned}
&\left.\int_{0}^{1} u^{r-1}(1-u)^{s-r-1}\left[1-t u^{\delta}(1-u)^{\omega}\right)\right]^{-2 a} E_{\lambda}[p u(1-u)] d u \\
&=\sum_{n=0}^{\infty} \frac{(2 a)_{n} t^{n}}{n !}{ }_{3} \Psi_{2}\left[\begin{array}{rr}
(r+\delta n, 1),(s-r+\omega n, 1),(1,1) ; & \\
(s+\delta n+\omega n, 2),(1, \lambda) ; & p
\end{array}\right]
\end{aligned}
$$

for $s>r>0, \lambda \geq 0, \delta, \omega \geq 0, \delta+\omega>0$

\section{Applications}

We derive some interesting results as applications of the integrals discussed in Section 2.

Example 4.1 First we take $\alpha_{1}=\alpha_{2}, \alpha=\beta>0$ and $x_{2}=x_{1} /\left(x_{1}-1\right)$ in (2.2) to find

$$
\begin{gathered}
I_{0,1, \lambda}^{\alpha, \alpha, 1}\left[\left(1-x_{1} t\right)^{-\alpha_{1}}\left(1-\frac{x_{1}}{x_{1}-1} t\right)^{-\alpha_{1}}, t(1-t) ; p\right] \\
=\sum_{m, n=0}^{\infty} \frac{(\alpha)_{m+n}\left(\alpha_{1}\right)_{m}\left(\alpha_{1}\right)_{n} x_{1}^{m}\left(\frac{x_{1}}{x_{1}-1}\right)^{n}}{(2 \alpha)_{m+n} m ! n !} \hat{\Psi}_{2}\left[\begin{array}{rr}
(\alpha, 1),(\alpha+m+n, 1),(1,1) ; & \\
(2 \alpha+m+n, 2),(1, \lambda) ; & p
\end{array}\right]
\end{gathered}
$$

for $\Re(\alpha)>0, \lambda \geq 0,\left|x_{1}\right|<1, \Re\left(x_{1}\right)<\frac{1}{2}$.

If $\alpha_{2}=0$ in (2.2), we obtain

$$
I_{0,1, \lambda}^{\alpha, \beta, 1}\left[\left(1-x_{1} t\right)^{-\alpha_{1}}, t(1-t) ; p\right]=\sum_{m=0}^{\infty} \frac{(\alpha)_{m}\left(\alpha_{1}\right)_{m} x_{1}^{m}}{(\alpha+\beta)_{m} m !}{ }_{3} \hat{\Psi}_{2}\left[\begin{array}{c}
(\alpha+m, 1),(\beta, 1),(1,1) ; \\
(\alpha+\beta+m, 2),(1, \lambda) ;
\end{array}\right]
$$


If, in addition, $\alpha_{1}=0$ we find

$$
I_{0,1, \lambda}^{\alpha, \beta}[1, t(1-t) ; p]={ }_{3} \hat{\Psi}_{2}\left[\begin{array}{cc}
(\alpha, 1),(\beta, 1),(1,1) ; & \\
(\alpha+\beta, 2),(1, \lambda) ;
\end{array}\right]
$$

which reduces to the form

$$
I_{0,1,1}^{\alpha, \beta, 1}[1, t(1-t) ; p]={ }_{2} F_{2}\left[\begin{array}{cc}
\alpha, \beta ; & \frac{p}{2}, \frac{\alpha+\beta+1}{2} ; \frac{1}{4}
\end{array}\right]
$$

when $\lambda=1$. The conditions applying to (4.2) - (4.4) are those appearing in Theorem 1 .

Example 4.2 If $x_{2}=x_{1} /\left(x_{1}-1\right)$ in (2.4), we obtain

$I_{0,1, \lambda}^{\alpha, \beta}\left[\frac{\left(1-x_{1} t\right)^{-\alpha_{1}-\alpha_{2}}}{1-x_{1}}, t(1-t) ; p\right]=\frac{\left(1-x_{1}\right)^{-1}}{B(\alpha, \beta)} \int_{0}^{1} t^{\alpha-1}(1-t)^{\beta-1}\left(1-x_{1} t\right)^{-\alpha_{1}-\alpha_{2}} E_{\lambda}[p t(1-t)] d t$.

Then replacing $\alpha_{1}$ by $\alpha_{1}+\alpha_{2}$ and $\alpha_{2}$ by 0 in the right-hand side of (2.4), we obtain

$$
\begin{aligned}
I_{0,1, \lambda}^{\alpha, \beta, 1}\left[\frac{\left(1-x_{1} t\right)^{-\alpha_{1}-\alpha_{2}}}{1-x_{1}}, t(1-t) ; p\right] \\
\quad=\frac{1}{1-x_{1}} \sum_{m=0}^{\infty} \frac{(\alpha)_{m}\left(\alpha_{1}+\alpha_{2}\right)_{m} x_{1}^{m}}{(\alpha+\beta)_{m} m !} \hat{\Psi}_{2}\left[\begin{array}{cc}
(\alpha+m, 1),(\beta, 1),(1,1) ; & \\
(\alpha+\beta+m, 2),(1, \lambda) ;
\end{array}\right]
\end{aligned}
$$

for $\Re(\alpha)>0, \Re(\beta)>0, \lambda \geq 0,\left|x_{1}\right|<1$. Alternatively, this result can be obtained directly from the above integral by series expansion of $E_{\lambda}[p t(1-t)]$ and use of the integral representation of the Gauss hypergeometric function in (1.6).

If $\lambda=1,(4.5)$ reduces to

$$
\begin{aligned}
& I_{0,1,1}^{\alpha, \beta, 1}\left[\frac{\left(1-x_{1} t\right)^{-\alpha_{1}-\alpha_{2}}}{1-x_{1}}, t(1-t) ; p\right] \\
& =\frac{1}{1-x_{1}} \sum_{m=0}^{\infty} \frac{(\alpha)_{m}\left(\alpha_{1}+\alpha_{2}\right)_{m} x_{1}^{m}}{(\alpha+\beta)_{m} m !}{ }_{2} F_{2}\left[\begin{array}{cc}
\alpha+m, \beta ; & p \\
\frac{\alpha+\beta+m}{2}, \frac{\alpha+\beta+m+1}{2} ; & 4
\end{array}\right]
\end{aligned}
$$

under the same conditions as (4.5).

Example 4.3 If $\gamma=-\alpha_{1}, u=-x_{1}, v=1, a=0$ and $b=1$ in (2.6), we obtain

$$
\begin{aligned}
& I_{0,1, \lambda}^{\alpha, \beta,-\alpha_{1}}\left[\left(1-x_{1} t\right), t(1-t) ; p\right] \\
&=\sum_{m=0}^{\infty} \frac{(\alpha)_{m}\left(\alpha_{1}\right)_{m}\left(-x_{1}\right)^{m}}{(\alpha+\beta)_{m} m !} 3_{3} \hat{\Psi}_{2}\left[\begin{array}{cc}
(\alpha+m, 1),(\beta, 1),(1,1) ; & \\
(\alpha+\beta+m, 2),(1, \lambda) ; & p
\end{array}\right]
\end{aligned}
$$

for $\Re(\alpha), \Re(\beta)>0, \lambda \geq 0,\left|x_{1}\right|<1$.

Example 4.4 If $\mu=\nu=0$ in (2.7), we obtain

$$
I_{a, b, \lambda}^{\alpha, \beta,-(\alpha+\beta)}\left[b-a, \frac{(t-a)(b-t)}{(b-a)^{2}} ; p\right]
$$




$$
=\frac{1}{(b-a)}{ }_{3} \hat{\Psi}_{2}\left[\begin{array}{cc}
(\alpha, 1),(\beta, 1),(1,1) ; \\
(\alpha+\beta, 2),(1, \lambda) ;
\end{array}\right]
$$

for $\Re(\alpha), \Re(\beta)>0, \lambda \geq 0, a \neq b$.

Example 4.5 Finally, if we take $\alpha=\beta, a=0$ and $b=1$ in (2.7), we obtain

$$
\begin{aligned}
I_{0,1, \lambda}^{\alpha, \alpha,-2 \alpha}[1+\nu t & \left.+\mu(1-t), \frac{t(1-t)}{(1+\nu t+\mu(1-t))^{2}} ; p\right] \\
= & {[(\nu+1)(\mu+1)]^{-\alpha}{ }_{3} \hat{\Psi}_{2}\left[\begin{array}{rc}
(\alpha, 1),(\alpha, 1),(1,1) ; & \left.\frac{p}{(\nu+1)(\mu+1)}\right]
\end{array}\right.}
\end{aligned}
$$

for $\Re(\alpha)>0, \lambda \geq 0$.

For $\lambda=1,(4.9)$ reduces to

$$
\begin{aligned}
I_{0,1,1}^{\alpha, \alpha,-2 \alpha}[1+\nu t+ & \left.\mu(1-t), \frac{t(1-t)}{(1+\nu t+\mu(1-t))^{2}} ; p\right] \\
= & {[(\nu+1)(\mu+1)]^{-\alpha}{ }_{1} F_{1}\left[\begin{array}{r}
\alpha ; \\
\alpha+\frac{1}{2} ;
\end{array} \frac{p}{4(\nu+1)(\mu+1)}\right] }
\end{aligned}
$$

for $\Re(\alpha)>0$.

\section{Discussion of integrals involving different kernels}

In all the integrals considered in this paper we have used the basic Mittag-Leffler function $E_{\alpha}(z)$ as kernel. There are many generalizations of this function that can be employed to extend the integrals in Sections 2, 3. For example, Prabhakar [16] introduced the function $E_{\alpha, \beta}^{\gamma}(z)$ in the form

$$
E_{\alpha, \beta}^{\gamma}(z)=\sum_{n=0}^{\infty} \frac{(\gamma)_{n}}{\Gamma(\alpha n+\beta)} \frac{z^{n}}{n !}, \quad(\alpha, \beta, \gamma \in \mathbf{C}, \Re(\alpha)>0, \Re(\beta)>0, \Re(\gamma)>0)
$$

When $\gamma=1$ this reduces to $E_{\alpha, \beta}(z)$ studied by Wiman [29]. A further generalization of the above function was given by Shukla and Prajapati [25]

$$
\begin{gathered}
E_{\alpha, \beta}^{\gamma, \delta}(z)=\sum_{n=0}^{\infty} \frac{(\gamma)_{\delta n}}{\Gamma(\alpha n+\beta)} \frac{z^{n}}{n !} \\
(\alpha, \beta, \gamma \in \mathbf{C}, \Re(\alpha)>0, \Re(\beta)>0, \Re(\gamma)>0, \delta \in(0,1) \cup \mathbf{N}),
\end{gathered}
$$

where $(\gamma)_{\delta n}=\Gamma(\gamma+\delta n) / \Gamma(\gamma)$. In 2018, Arshad et al. [1] introduced a new extension of the Mittag-Leffler function given by

$$
\begin{gathered}
E_{\alpha, \beta}^{\gamma, c ; \gamma, \rho}(z ; p)=\sum_{n=0}^{\infty} \frac{B_{p}^{\lambda, \rho}(\gamma+n, c-\gamma)}{B(\gamma, c-\gamma)} \frac{(c)_{n}}{\Gamma(\alpha n+\beta)} \frac{z^{n}}{n !} \\
\left(p \in \mathbf{R}_{0}^{+} ; \Re(c)>\Re(\gamma)>0 ; \Re(\alpha)>0 ; \rho \in \mathbf{C} \backslash \mathbf{Z}_{0}^{-}\right),
\end{gathered}
$$

where $B_{p}^{\lambda, \rho}$ is ... Other generalizations have been considered in $[20,21,24]$. 
Kiryakova [10, p. 244] defined the multi-index ( $m$-tuple) Mittag-Leffler function for integer $m \geq 1$ defined by

$$
E_{\left(\frac{1}{\rho_{m}}\right)\left(\mu_{m}\right)}(z)=\sum_{n=0}^{\infty} \frac{z^{n}}{\Gamma\left(\mu_{1}+\frac{n}{\rho_{1}}\right) \ldots \Gamma\left(\mu_{m}+\frac{n}{\rho_{m}}\right)}
$$

where the parameters $\rho_{1}, \rho_{2}, \ldots, \rho_{m}>0$ and $\mu_{1}, \mu_{2}, \ldots, \mu_{m}$ are arbitrary real numbers. The integral analogous to that in (2.1) involving the multi-index Mittag-Leffler function is

$$
I_{a, b,\left(\frac{1}{\rho_{q}}\right),\left(\mu_{q}\right)}^{\alpha, \beta, \gamma}[\chi(t), \xi(t) ; p]=\frac{1}{B(\alpha, \beta)} \int_{a}^{b}(t-a)^{\alpha-1}(b-t)^{\beta-1}[\chi(t)]^{\gamma} E_{\left(\frac{1}{\rho_{q}}\right),\left(\mu_{q}\right)}[p \xi(t)] d t
$$

for integer $q \geq 1$, which can be evaluated for some specific functions $\chi(t)$ and $\xi(t)$.

For the values of $a, b, \gamma, \xi(t)$ and $\chi(t)$ chosen in Theorem 1 , the above integral can be expressed in the following form

$$
\left.\begin{array}{r}
I_{0,1,\left(\frac{1}{\rho_{q}}\right),\left(\mu_{q}\right)}^{\alpha, \beta, 1}\left[\left(1-x_{1} t\right)^{-\alpha_{1}}\left(1-x_{2} t\right)^{-\alpha_{2}}, t(1-t) ; p\right]=\frac{1}{\prod_{r=1}^{q} \Gamma\left(\mu_{r}\right)} \sum_{m, n=0}^{\infty} \frac{(\alpha)_{m+n}\left(\alpha_{1}\right)_{m}\left(\alpha_{2}\right)_{n} x_{1}^{m} x_{2}^{n}}{(\alpha+\beta)_{m+n} m ! n !} \\
\times{ }_{3} \hat{\Psi}_{q+1}\left[\begin{array}{c}
(\alpha+m+n, 1),(\beta, 1),(1,1) ; \\
p
\end{array}\right] \\
\left(\mu_{1}, \frac{1}{\rho_{1}}\right), \ldots,\left(\mu_{q}, \frac{1}{\rho_{q}}\right),(\alpha+\beta+m+n, 2) ;
\end{array}\right]
$$

for $\Re(\alpha), \Re(\beta)>0, \rho_{1}, \ldots, \rho_{q}>0, \mu_{1}, \ldots, \mu_{q}$ arbitrary real numbers and $\left|x_{1}\right|,\left|x_{2}\right|<1$. We recall that $\hat{\Psi}_{q}$ is the normalized Wright function defined in (1.3). In the case $q=1, \mu_{1}=1$, $\rho_{1}=1 / \lambda$, the right-hand side of (5.3) reduces to $(2.2)$.

Let the generating function $G\left(x, t u^{\delta}(1-u)^{\omega}\right)$ be uniformly convergent under the conditions $u \in(0,1), \delta, \omega \geq 0$ and $\delta+\omega>0$, with $G(x, t)$ defined by (3.1). Then

$$
\begin{array}{rl}
\int_{0}^{1} u^{r-1}(1-u)^{s-r-1} & G\left(x, t u^{\delta}(1-u)^{\omega}\right) E_{\left(\frac{1}{\rho_{q}}\right),\left(\mu_{q}\right)}[p u(1-u)] d u \\
=\sum_{n=0}^{\infty} c_{n} g_{n}(x) t^{n}{ }_{3} \Psi_{q+1}\left[\begin{array}{cc}
(r+\delta n, 1),(s-r+\omega n, 1),(1,1) ; & p \\
\left(\mu_{1}, \frac{1}{\rho_{1}}\right), \ldots,\left(\mu_{q}, \frac{1}{\rho_{q}}\right),(s+\delta n+\omega n, 2) ;
\end{array}\right]
\end{array}
$$

for $s>r>0, \rho_{1}, \ldots, \rho_{q}>0, \mu_{1}, \ldots, \mu_{q}$ arbitrary real numbers and $p \in \mathbf{C}$. We recall that ${ }_{p} \Psi_{q}$ is the un-normalized Wright function defined in (1.1).

\section{Concluding remarks}

In this paper, we have evaluated some definite Euler-type integrals. We observe that these evaluations are obtained in terms of the Wright hypergeometric function ${ }_{3} \Psi_{2}$. In addition, we have derived a theorem and applied it to obtain evaluations of these integrals related to ${ }_{3} \Psi_{2}$. Further, we remark that the results presented in this paper can be extended to the multi-variable case.

For this purpose, we employ the following integral representation [9, p. 965(20)] for Lauricella's multiple hypergeometric series in $n$-variables $F_{D}^{(n)}[28]$

$$
\begin{gathered}
\int_{0}^{1} t^{\alpha-1}(1-t)^{\beta-1} \prod_{i=1}^{n}\left(1-x_{i} t\right)^{-\alpha_{i}} d t=B(\alpha, \beta) F_{D}^{(n)}\left(\alpha, \alpha_{1}, \ldots, \alpha_{n} ; \alpha+\beta ; x_{1}, \ldots, x_{n}\right) \\
\left(\Re(\alpha), \Re(\beta)>0, \max \left\{\left|x_{1}\right|, \ldots,\left|x_{n}\right|\right\}<1\right) .
\end{gathered}
$$


If we let $\phi(t)=\prod_{i=1}^{n}\left(1-x_{i} t\right)^{-\alpha_{i}}, \xi(t)=t(1-t), a=0$ and $b=\gamma=1$ in (2.1), and use (6.1), we obtain upon simplification

$$
\begin{aligned}
I_{0,1, \lambda}^{\alpha, \beta, 1}\left[\prod_{i=1}^{n}\left(1-x_{i} t\right)^{-\alpha_{i}}, t(1-t) ; p\right] & =\frac{1}{B(\alpha, \beta)} \sum_{m_{1}, \ldots, m_{n}=0}^{\infty} \frac{\left(\alpha_{1}\right)_{m_{1}} \ldots\left(\alpha_{n}\right)_{m_{n}} x_{1}^{m_{1}} \ldots x_{n}^{m_{n}}}{m_{1} ! \ldots m_{n} !} \\
\times{ }_{3} \Psi_{2} & {\left[\begin{array}{c}
\left(\alpha+m_{1}+\ldots+m_{n}, 1\right),(\beta, 1),(1,1) ; \\
\left(\alpha+\beta+m_{1}+\ldots+m_{n}, 2\right),(1, \lambda) ;
\end{array}\right] }
\end{aligned}
$$

for $\Re(\alpha), \Re(\beta)>0, \lambda \geq 0, \max \left\{\left|x_{1}\right|, \ldots,\left|x_{n}\right|\right\}<1$. We observe that (6.2) reduces to (2.2) for $\alpha_{3}=\alpha_{4}=\ldots=\alpha_{n}=0$.

As a consequence, we can extend Theorem 5 as follows:

Theorem 6. Let us take the conditions for $G(x, t)$ defined by (3.1) to be the same as in Theorem 5. Then we have

$$
\begin{aligned}
\int_{0}^{1} u^{r-1}(1-u)^{s-r-1} \prod_{i=1}^{j}(1- & \left.x_{i} u\right)^{-\alpha_{i}} G\left(x, t u^{\delta}(1-u)^{\omega}\right) E_{\lambda}[p u(1-u)] d u \\
& =\sum_{n, m_{1}, \ldots, m_{j}=0}^{\infty} c_{n} g_{n}(x) t^{n} \times \frac{\left(\alpha_{1}\right)_{m_{1}} \ldots\left(\alpha_{n}\right)_{m_{n}} x_{1}^{m_{1}} \ldots x_{n}^{m_{n}}}{m_{1} ! \ldots m_{n} !} \\
& \times{ }_{3} \Psi_{2}\left[\begin{array}{r}
\left(r+\delta n+m_{1}+\ldots+m_{j}, 1\right),(s-r+\omega n, 1),(1,1) ; \quad \\
\left(s+\delta n+\omega n+m_{1}+\ldots+m_{j}, 2\right),(1, \lambda) ;
\end{array}\right]
\end{aligned}
$$

for $\Re(\delta)>\Re(\omega)>0, \lambda \geq 0, \delta, \omega>0, \delta+\omega>0, p \in \mathbf{C}$.

The proof of this theorem is similar to that of Theorem 5 and will be omitted.

Corollary 2. By considering Theorem 6 then we find, when $\lambda=1$,

$$
\begin{aligned}
\int_{0}^{1} u^{r-1}(1-u)^{s-r-1} \prod_{i=1}^{j}(1- & \left.x_{i} u\right)^{-\alpha_{i}} G\left(x, t u^{\delta}(1-u)^{\omega}\right) E_{\lambda}[p u(1-u)] d u \\
& =\sum_{n, m_{1}, \ldots, m_{j}=0}^{\infty} c_{n} g_{n}(x) t^{n} \frac{\left(\alpha_{1}\right)_{m_{1}} \ldots\left(\alpha_{n}\right)_{m_{n}} x_{1}^{m_{1}} \ldots x_{n}^{m_{n}}}{m_{1} ! \ldots m_{n} !} \\
& \times{ }_{2} \Psi_{1}\left[\begin{array}{rr}
\left(r+\delta n+m_{1}+\ldots+m_{j}, 1\right),(s-r+\omega n, 1) ; & p \\
\left(s+\delta n+\omega n+m_{1}+\ldots+m_{j}, 2\right) ;
\end{array}\right]
\end{aligned}
$$

for $\Re(\delta)>\Re(\omega)>0 ; \delta, \omega>0 ; \delta+\omega>0 ; p \in \mathbf{C}$.

It is clear that by applying Theorem 6 and Corollary 2 to the generating functions $G(x, t)$ defined by (3.5) and (3.7), together with some other generating functions, we may obtain many interesting evaluations.

\section{Acknowledgement}

The authors thank the constructive comments and suggestions by theneditor and anonymous referees, which have contributed to the improvement of the presentation of this paper. 


\section{References}

[1] M. Arshad, J. Choi, S. Mubeen, K.S. Nisar and G. Rahman, A new extention of the Mittag-Leffler function, Commun. Korean Math. Soc., 33(2) (2018), 549-560.

[2] W.N. Bailey, Generalized Hypergeometric Series, Cambridge University Press, Cambridge, 1935; Reprinted by Stechert Hafner, New York, 1964.

[3] M.A. Chaudhry, A. Qadir, M. Raflque and S.M. Zubair, Extension of Euler's Beta function, J. Comput. Appl. Math. 78 (1997), 19-32.

[4] M.A. Chaudhry, A. Qadir, H.M. Srivastava and R.B. Paris, Extended hypergeometric and confluent hypergeometric functions, Appl. Math. Comput. 159 (2004), 589-602.

[5] M.A. Chaudhry and S.M. Zubair, Generalized incomplete gamma functions with applications, J. Comput. Appl. Math. 55 (1994), 99-124.

[6] M.A. Chaudhry and S.M. Zubair, On an extension of generalized incomplete gamma functions with applications, J. Austral. Math. Soc. Ser. B 37 (1996), 392-405.

[7] C. Fox, The asymptotic expansion of generalized hypergeometric functions, Proc. London Math. Soc. (2) 27 (1928), 389-400.

[8] R. Gorenflo, A.A Kilbas, F. Mainardi and S.V. Rogosin, Mittag-Leffler Functions, Related Topics and Applications, Springer, Berlin, 2014.

[9] M.E.H. Ismail and J. Pitman, Algebraic evaluations of some Euler integrals, duplication formulae for Appells hypergeometric function $F_{1}$, and Brownian variations, Canad. J. Math. 52 (5) (2000), 961-981.

[10] V.S. Kiryakova, Multiple (multiindex) Mittag-Leffler functions and relations to generalized fractional calculus, J. Comp. Appl. Math., 118(2000), 241-259.

[11] G.M. Mittag-Leffler, Sur la nouvelle fonction $E_{\alpha}(x)$, C. R. Acad. Sci. Paris 137 (1903), 554-558.

[12] G.M. Mittag-Leffler, Sur la répresentation analytique d'une branche uniforme d'une fonction monogène, Acta Math. 29 (1905), 101-181.

[13] F.W.J. Olver, D.W. Lozier, R.F. Boisvert and C.W. Clark (eds.). NIST Handbook of Mathematical Functions. Cambridge University Press, Cambridge, 2010.

[14] E. Özergin, M.A. Özarslan and A. Altin, Extension of gamma, beta and hypergeometric functions, J. Comp. Appl. Math. 235 (2011), 4601-4610.

[15] J. Pitman, Brownian motion, bridge, excursion and meander characterized by sampling at independent uniform times, Electron. J. Probab. 11(4) (1999), 1-33.

[16] T.R. Prabhakar, A singular integral equation with a generalized Mittag-Leffler function of the kernel, Yokohana. Math. J.,19 7-15 (1971).

[17] A.P. Prudnikov, Yu.A. Brychkov and O.I. Marichev, Integrals and Series, Vol. I, Gordon and Breach Science Publishers, New York 1981.

[18] A.P. Prudnikov, Yu.A. Brychkov and O.I. Marichev, Integrals and Series, Vol. III, Gordon and Breach Science Publishers, New York 1990.

[19] E.D. Rainville, Special Functions, Macmillan Company, New York, 1960; Reprinted by Chelsea Publishing Company, Bronx, New York, 1971.

[20] T.O. Salim, Some properties relating to the generalized Mittag-Leffler function , Adv. Appl. Math. Anal.,4 21-30 (2009).

[21] T.O. Salim and W. Faraj Ahmad, A generalization of Mittag-Leffler function and integral operator associated with fractional calculus, J. Fract. Cal. Appl.,3(5) 1-13 (2012).

[22] M. Shadab and J. Choi, Extensions of Appell and Lauricella hypergeometric Functions, Far East J. Math. Sci, 102(6) (2017), 1301-1317.

[23] M. Shadab, S. Jabee and J. Choi, An extended beta function and its applications, Far East J. Math. Sci, 103(1) (2018), 235-251. 
[24] M.A. Khan and S. Ahmad, On some properties of the generalized Mittag-Leffler function, Springer Plus,2 (2013), 1-9.

[25] A.K. Shukla and J.C. Prajapati, On a generalisation of Mittag-Leffler function and its properties, J. Math Anal and Appl., 336 797-811 (2007).

[26] H.M. Srivastava and J. Choi, Zeta and q-Zeta Functions and Associated Series and Integrals, Elsevier Science Publishers, Amsterdam, London and New York, 2012.

[27] H.M. Srivastava and P.W. Karlsson, Multiple Gaussian hypergeometric Series, Ellis Horwood Series: Mathematics and its Applications. Ellis Horwood Ltd., Chichester; Halsted Press [John Wiley \& Sons, Inc.], New York, 1985.

[28] H.M. Srivastava and H.L. Manocha, A Treatise on Generating Functions, Halsted Press (Ellis Horwood Limited, Chichester), John Wiley and Sons, New York, Chichester, Brisbane and Toronto, 1984.

[29] A. Wiman, Uber den Fundamentalsatz in der Theorie der Funktionen $E_{\alpha}(x)$, Acta Math.,29 191-207 (1905).

[30] E.M. Wright, The asymptotic expansion of the generalized hypergeometric functions, J. London. Math. Soc. 10 (1935), 286-293.

[31] E.M. Wright, The asymptotic expansion of integral functions defined by Taylor series, Philos. Trans. Roy. Soc. London, Ser. A. 238 (1940), 423-451. 